\title{
INFLUENCIA DE LA POLÍTICA EN LOS CUIDADOS A INMIGRANTES Y GRUPOS DESFAVORECIDOS
}

\author{
Blázquez Rodríguez, $\mathbf{M}^{\mathbf{a}}$. Isabel \\ Diplomada Universitaria en Enfermería. Licenciada en Antropología Social y \\ Cultural. Diploma de Especialización en Promoción de Salud. Investigadora del \\ Instituto de Salud Carlos III. Madrid
}

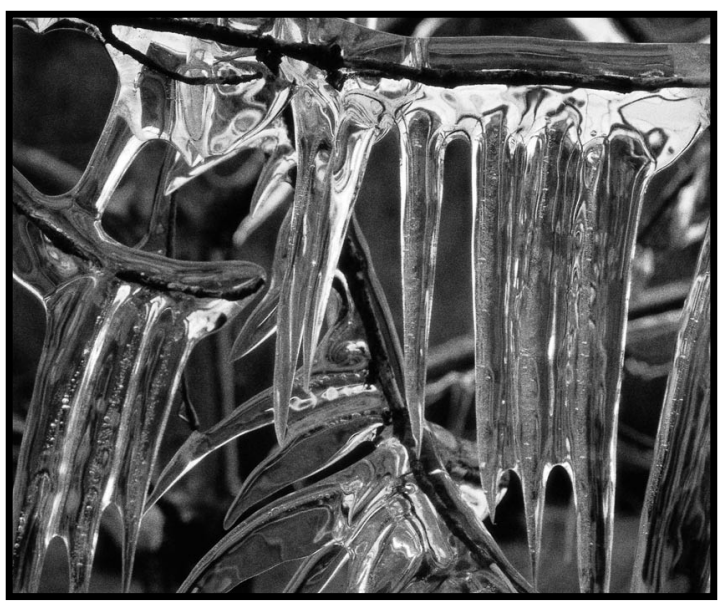

\section{INFLUENCE OF POLITICS ON CARE OF IMMIGRANTS AND VULNERABLE GROUPS}

\begin{abstract}
Immigration policies are to a great extent determined by established legislation. The requirements that appoint the right to health protection and the use of health services. have changed during the past years.

The ideas hereby included come from my experience in participative research-action with immigrants communities in the Escuela Nacional de Sanidad, the objective being to give data and analise the different policies, based on health legislation or others.
\end{abstract}

Key words: immigration policies, vulnerable groups

\section{RESUMEN}

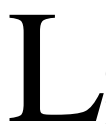

as políticas en materia de extranjería vienen determinadas en gran medida por las legislaciones que se establecen. Los requisitos que han establecido para la atención han variado en los últimos años y determinan el derecho a la protección de la salud y la utilización de los servicios sanitarios.

Las ideas que aquí se plantean parte de mi experiencia en la investigación-acción participativa con comunidades inmigradas en la Escuela Nacional de Sanidad, el objetivo es aportar datos y analizar las diferentes políticas, centradas en las legislaciones, tanto sanitarias como de otro orden.

Palabras clave: política de inmigración, grupos desfavorecidos.

\section{INTRODUCCIÓN}

Las políticas en materia de extranjería vienen determinadas en gran medida por las legislaciones que se establezcan. Así en estas legislaciones se regula la accesibilidad a la atención sanitaria por parte de la población extranjera. Los requisitos que han establecido para la atención han variado en los últimos años y determinan el derecho a la protección de la salud y la utilización de los servicios sanitarios. Además estas legislaciones regulan otros aspectos de la vida ordinaria de la persona extranjera que influyen en su nivel de salud como el acceso al trabajo, a la vivienda, a la educación,...

Las ideas que aquí se plantean parte de mi experiencia en la investigación-acción participativa con comunidades inmigradas en la Escuela Nacional de Sanidad, sin considerarme experta en este asunto, si quisiera aportar algunos datos y análisis que nos muestran diferentes políticas, centrándome en las legislaciones, tanto sanitarias como de otro orden, que afectan a los grupos inmigrantes pero también a la población autóctona especialmente a aquellos grupos más desfavorecidos en su derecho a la protección a la salud. La convergencia de estas variadas políticas nos dibujan el panorama 
de cómo se ha definido la atención sanitaria y cómo esta condicionada por una serie de factores para una parte de nuestra población.

Finalmente lo que se pone en cuestionamiento es la estructura de nuestro estado de bienestar que debe ser el garante del derecho a la protección de la salud y del nivel de calidad de vida de sus ciudadanos/as.

\section{REVISIÓN DE LAS LEGISLACIONES}

Haciendo un breve recordatorio de las legislaciones encontramos que el primer ordenamiento jurídico en materia de extranjería se publica en 1985 (BOE núm. 158), que sólo contemplaba el derecho a la protección de la salud de aquellos extranjeros regularizados. Pero es la ley 4/2000 en su artículo 12, la que expresa que tienen derecho a la asistencia sanitaria aquellos extranjeros que se encuentren en España en alguna de las siguientes situaciones:

Los extranjeros que se encuentren en España inscritos en el padrón del municipio en el que residan habitualmente tienen derecho a la asistencia sanitaria en las mismas condiciones que los españoles.

Los extranjeros que se encuentren en España tienen derecho a la asistencia sanitaria pública de urgencia ante la contracción de enfermedades graves o accidentes, cualquiera que sea su causa, y a la continuidad de dicha atención hasta la situación de alta médica.

1. Los extranjeros menores de dieciocho años que se encuentren en España tienen derecho a la asistencia sanitaria en las mismas condiciones que los españoles.

2. Las extranjeras embarazadas que se encuentren en España tendrán derecho a la asistencia sanitaria durante el embarazo, parto y posparto.

En todas estas legislaciones lo que se detecta es que establecen la jerarquización de derechos, la consideración del inmigrante como mano de obra y la vinculación entre trabajo y residencia, y esta última se vuelve más inespecífica y abstracta, lo que da lugar a una mayor arbitrariedad.

En esta ley 4/2000 aparece la inscripción en el padrón como el mecanismo facilitador de la atención de aquellos extranjeros que no estén regularizados. Después del empadronamiento la persona extranjera deberá realizar otro trámite administrativo que consiste en la tarjeta sanitaria. Para ello debe aportar una acreditación de insuficiencia de recursos económicos, el certificado de empadronamiento, pasaporte y formularios F1 y F6. El formulario F6 consiste en la solicitud de reconocimiento del derecho a la asistencia sanitaria y el F1 en la solicitud de emisión de la tarjeta sanitaria.

De todo este corpus legislativo quisiera comentar algunos aspectos que condicionan el disfrute de este derecho a la protección de la salud de la población de origen extranjero:

El primer efecto que se puede evidenciar con la apertura de la Ley 4/2000 en materia de derecho a la asistencia sanitaria, es que ha tenido efectos positivos inmediatos en la normalización de la atención en salud para los inmigrantes. Si analizamos los datos de altas en tarjetas sanitarias en la Comunidad de Madrid, nos muestran que antes de esta ley había sólo 11.707 tarjetas sanitarias, y a 1 de enero del 2002 había 358.616. Este crecimiento tan rápido del $41.51 \%$, desde enero a octubre del 2002 nos verifica la necesidad que había de una ley que facilitase la atención sanitaria de la población inmigrante.

En segundo lugar, la ley contempla que la atención sanitaria esta supeditada a la inscripción en el padrón. Por lo que todas aquellas personas que no puedan empadronarse por diferentes circunstancias quedarían ausentes de derecho. Las dificultades para empadronarse pueden ser porque carecen de documentación identificativa o porque son itinerantes por trabajo, carecen de vivienda estable, en su vivienda hay muchas personas empadronadas, o la primera persona empadronada en la vivienda no le autoriza el empadronamiento,... El documento identificativo puede ser bien el documento oficial expedido por las autoridades españolas (tarjeta de extranjero) o bien el expedido por las autoridades del país de origen, sin embargo hay algunas personas inmigrantes que carecen de ambos documentos. La mayor parte de estos extranjeros no empadronados son los que se encuentra en unas condiciones de vida y exclusión social que ponen su salud en una situación de mayor vulnerabilidad (Castillo, 2002) por lo que necesitarían poder entrar en los circuitos preventivos y asistenciales. 
El padrón se convierte en un instrumento para la adquisición de derechos que es normativizado por las administraciones locales, dándose una enorme arbitrariedad entre municipios para su tramitación y concesión. Por lo que se produce una desigual accesibilidad en función de las áreas geográficas. Además en la recién estrenada ley 14/2003 se expresa que los datos del Padrón Municipal se cederán a otras Administraciones Públicas que lo soliciten, sin consentimiento previo del inmigrante: "La Dirección General de la Policía accederá a los datos de inscripción padronal de los extranjeros existentes en los Padrones Municipales”. Además la reforma permite saber quienes son "regulares" y quienes "irregulares", mediante los datos de identificación. Y exige que el padrón sea renovado cada dos años aunque no haya modificaciones. Por lo que el padrón queda concebido como instrumento de control administrativo o policial. Los efectos que hemos percibido de esta medida es que desalienta a un gran número de inmigrantes a su empadronamiento ya que sienten que esto es una medida de control y como las medidas de expulsión se endurecen, prefieren no empadronarse, aunque ello implique no poder optar a estos derechos que el trámite confiere como el de atención sanitaria.

Todo ello produce que el número de irregulares se presente como un grupo cada vez más numeroso e invisibilizado sin acceso a los servicios de salud. Según el Anuario Estadístico Extranjería a 31 de junio de 2003 (INSALUD), el número de personas extranjeras es de 1.448.671, y según el INE con los datos del padrón esta cifra asciende a 2,5 millones de extranjeros, ya que se incluye a los que no tienen tarjeta o permiso de residencia, de tal forma que empadronados o extranjeros no regularizados habría 1.051 .329 personas. Siendo esta cifra sólo la punta del iceberg que esconde detrás de sí a otras personas que no son registradas.

La existencia de un gran número de personas en situación irregular también puede ser conocida a partir de los datos ofrecidos por la Delegación del Gobierno para la Inmigración, como resultado de las regularizaciones extraordinarias realizadas en los dos últimos procesos (2000 y 2001). El Real Decreto 239/2000 (BOE NÚM. 43) establece el procedimiento para la regularización de extranje- ros prevista en la ley 4/2000 y da lugar al proceso de regulación iniciado en el 2000.

Posteriormente el Real Decreto 142/2001(BOE num 44) establece el proceso de regularización extraordinario del 2001. Según esta información se otorgó documentación a 334.882 inmigrantes desde marzo de 2000 a diciembre de 2001, de un total de 615.000 solicitudes presentadas. Lo que quiere decir que el volumen de extranjeros sin regularizar era antes de los procedimientos muy elevado, y que sigue siéndolo después, ya que a la cifra de denegaciones ha de añadirse la de nuevas entradas producidas en este período hasta la actualidad.

Otras regulaciones que afectan a sanidad y debemos conocer es lo dispuesto en Reglamentos y Acuerdos Sanitarios en materia de sanidad exterior (Real Decreto 1418/1986 de 13 de junio). Sobre los requisitos sanitarios que deberán presentar todas las personas a su entrada en el territorio español: "cuando así lo determine el Ministerio del Interior (...) deberán presentar un certificado sanitario expedido en el país de procedencia por los servicios médicos que designe la Misión Diplomática u Oficina Consular española o someterse a reconocimiento médico por parte de los servicios sanitarios españoles competentes a su llegada en la frontera con el fin de acreditar que no padece ninguna de las enfermedades cuarentenales (...)". La exigencia de un certificado médico ha supuesto en algunos casos una ampliación del diagnóstico de enfermedades cuarentenales a otras enfermedades con la violación del principio de consentimiento informado y confidencialidad, derecho básico en las relaciones profesional/ usuario. Además este trámite se ha utilizado en ocasiones como una medida para controlar y regular la entrada de inmigrantes y por los empresarios para realizar un contrato laboral.

Finalmente se habla de razones humanitarias para solventar aquellas situaciones que requieran regularización, y no se ajusten a lo descrito en el texto legislativo. Sin embargo parece erróneo pensar que estas situaciones puedan resolverse de este modo. Esto podría producir como ha ocurrido en países cercanos (Francia) una terrible paradoja de que solo la enfermedad, por razón humanitaria, confiere derechos. Cabría preguntarnos qué ocurre con las enfermedades y problemas de salud no urgentes 
en la población inmigrante irregular ¿se dejan a la voluntariedad de profesionales y servicios o se acogen al régimen de razones humanitarias?

Por todo ello, las exigencias legislativas convierten el aspecto de la documentación en fundamental para la vida del inmigrante en España, y en consecuencia, la eficacia de su trámite deja de ser deseable para pasar a ser indispensable, ya que de ella dependerá la posibilidad y condiciones de estancia de esas personas.

\section{INFLUENCIA DE LAS POLÍTICAS EN LA SALUD}

En la Escuela Nacional de Sanidad, inicialmente dentro del Departamento de Salud Internacional se empieza en 1997 una línea de investigación hacia el binomio salud-inmigración, con objeto de conocer cual era el perfil socio sanitario de este colectivo, su acceso a los servicios de salud (cobertura sanitaria,) sus problemas de salud,... En esos años es cuando en nuestro país la población inmigrante estaba aumentando considerablemente y sobre todo aquella procedente de países en desarrollo. Esta mayor relevancia de conocimiento de estos grupos sociales desfavorecidos ha servido para señalar las desigualdades en salud todavía hoy presentes en el seno de nuestras sociedades de bienestar y desarrollo (Carballo, 1996).

De este modo tenemos que hablar de los determinantes que influyen en la salud además de considerar aquellos derivados del estilo de vida que pueden poner en peligro la salud, hay que considerar aquellos derivados de las condiciones externas que soportan por su condición de inmigrantes. Resulta difícil hacer una lista de todos ellos pero a grandes rasgos podemos hablar en primer lugar del proceso migratorio, de la situación de irregularidad, los aspectos socio-económicos (relacionados con la dificultad de encontrar un trabajo y de que este cumpla unos mínimos que garanticen la dignidad de la persona), el acceso a la educación, a la reagrupación familiar, los prejuicios y los obstáculos para la integración social,... Como dice Mary Shaw (1999): "Junto a los factores culturales existen otros como la pobreza, la privación y la exclusión social que influyen en un estado de salud negativo, incrementándose este hecho entre las minorías étnicas y colectivos de inmigrantes cuan- do se añaden los efectos de la xenofobia y del prejuicio social".

Destaca el sistema sanitario como determinantes de la salud ya que encontramos que aunque se de la accesibilidad a los servicios a través del reconocimiento legal; encontramos otros factores de índole económica, cultural, política,... que producen que el proceso de atención sanitaria sea interrumpido. Por ello hablamos de barreras (Castillo, 2002) en el acceso y utilización de la atención sanitaria. Algunas de estas barreras aparecen también con otros grupos en situación de desigualdad como pueden ser minorías sociales y grupos étnicos. A su vez estas barreras proceden tanto de:

- La propia población inmigrante, como son: el idioma y los problemas de comunicación, miedos, desconfianzas y rechazos, obstáculos culturales, religiosos y morales, creencias y usos de la medicina tradicional y natural, concepto de salud, cuidados y enfermedad,...

- Los/as profesionales sanitarios, como son: la percepción de abuso, sobrecarga asistencial, desconocimiento de la cultura, de la legislación y recursos sociales, estereotipos sobre inmigración, estigmatización:

- Los servicios de salud, como son: la burocracia, los requisitos administrativos, los sistemas de gestión de las consultas, la concentración de inmigrantes en los cupos de algunos profesionales, los sistemas de registros y citas, los horarios, la descoordinación de los servicios, pocos programas y materiales educativos adaptados culturalmente,...

\section{AUTOPERCEPCIÓN DE LA SALUD DE LA POBLACIÓN INMIGRANTE}

Por parte de la población inmigrante sabemos (Proyecto SEPY 98) que su salud no es una prioridad, su importancia es en la medida en que se asocia a la capacidad y la autonomía para desempeñar un trabajo. Por lo que trabajo y salud están recíprocamente relacionados y el desempleo influye en una mala salud, y la perdida de salud lleva al desempleo.

Pero además cuando se habla de salud en las entrevistas con los/as inmigrantes aparece un "discurso de la queja" por psicosomatización del malestar social. En la salud y enfermedad es donde más relevante y patente se hace la relación entre el 
cuerpo con lo social, lo espiritual y lo psicológico. El cuerpo somatiza (Atxotegui, 2000), manifiesta con disfunciones orgánicas las frustraciones y conflictos cuya génesis se debe a una situación de estrés prolongada, carencias afectivas, fracaso de expectativas, experiencias de rechazo, escasez económica, inactividad, etc. Por lo que ante los signos y síntomas que manifiesta el sujeto, hemos de analizar los procesos no sólo biológicos, sino también sociales, políticos, económicos, es decir, correlacionar lo que siente con las circunstancias en las que se encuentra. Cabe preguntar si cierta morbilidad asociada a grupos como mujeres y depresión, jóvenes y trastornos de la alimentación, inmigración y psico somatizaciones, no son el resultado de factores estructurales en los que se encuentran estos grupos. Además mientras estas condiciones no se modifiquen el cuerpo estará avocado a la enfermedad y al mantenerse pueden derivar en problemas de salud crónicos.

\section{RETOS PARA EL FUTURO}

1. Visibilización de la situación de salud de estos grupos como implica por ejemplo el ajuste de los sistemas de registro epidemiológico, de morbilidad,... desglosados por una variable que recoja su condición de inmigrados como país de origen.

2. Políticas sanitarias adaptadas a las características de esta población. Lo que implica valorar si existen realmente diferencias entre los inmigrantes y otros grupos desfavorecidos socialmente. Es decir, evitar un discurso diferencialista que resaltando las diferencias culturales oculte otros factores como los socioeconómicos. Si realmente se dan peculiaridades que requieran intervenciones específicas como sucede con los recién llegados. Otras intervenciones son las dirigidas a las barreras de acceso a la información y a los servicios de salud, junto con la dificultad para comprender los mensajes preventivos por el desconocimiento del idioma, por la utilización de un lenguaje distinto, por los diferentes valores y significados, por los aspectos culturales especialmente los que hacen alusión a la sexualidad o a las relaciones entre hombres y mujeres,...

3. Abordaje intersectorial de la salud: hay que comenzar, por dar respuesta a la realidad de la inmigración en sus múltiples facetas: medidas legislati- vas, administrativas, políticas, económicas y sociales para que los nuevos trabajadores/as y sus familias sean miembros de pleno derecho de la sociedad y mejoren sus niveles de salud y calidad de vida.

4. Establecer políticas públicas de previsión e inversión que eviten un sistema sanitario sobrecargado lo que conlleva dotar de recursos humanos, técnicos y materiales, que redundaría tanto en una mejora de la calidad asistencial como en la satisfacción de los diferentes usuarios/as.

5. Reclamación de políticas de discriminación positiva que no han de contemplarse como exclusivas para la población inmigrante sino que deberían ser aplicadas en general para facilitar el acceso a determinados recursos a los grupos más desfavorecidos socialmente como vivienda, becas,...

Por ejemplo una medida discriminativa positiva en la ley es que se puede obtener la autorización de residencia independiente, cuando "el cónyuge" (marido ó mujer) sea judicialmente declarado-a víctima de violencia doméstica, en virtud de una orden de protección judicialmente dictada a favor de la víctima cónyuge. Aunque no se concede idéntico trato a hijos/as, etc..., que sean víctimas de violencia doméstica, y tampoco se da autorización para trabajar.

6. Fomentar políticas de integración intercultural, que garanticen el respeto a la diversidad y así evitar que se les vea como un motivo de perturbación que suponga la marginalización y exclusión social. Esto implica facilitar una atención sanitaria transcultural (valorar qué tipo y para qué las figuras de mediación y la formación en competencia intercultural).

7. Reforzar Atención Primaria: si la población que emigra es joven y sana, las principales necesidades y demandas de salud giran entorno a la promoción de salud y prevención de enfermedades (Blázquez 2003).

8. Necesidad de políticas inclusivas de participación de las comunidades inmigradas en la prevención de enfermedades y en la promoción de su salud.

9. Evitar crear grupos de riesgo asociados a los inmigrantes por ser procedentes de lugares de origen y culturas diferentes, y esto por varias razones, entre ellas las de no fomentar actitudes de marginación, rechazo y estigmatización. 


\section{ALGUNAS NOTAS FINALES PARA EL DEBATE}

En la medida que dispongamos de un concepto de salud más amplio, que depende de factores de diversa índole, apostaremos por mejorar el funcionamiento de nuestra sociedad ya que la calidad de vida y la salud de sus ciudadanos/as estarán directamente relacionadas con múltiples y diversas intervenciones. Por ello quisiera plantear algunos datos de las políticas del estado de bienestar de nuestro país que nos orientan hacía dónde se dirigen los intereses en salud, y de esa forma podremos entender la viabilidad de todas estas propuestas dirigidas a los grupos más desfavorecidos socialmente:

Desde 1991 hay una política de recortes sanitarios públicos que tiene su origen en las políticas neoliberales que pretenden el déficit cero. El gasto en España en Protección Social, dónde entra la salud, es de los más bajos de Europa. Dentro del gasto sanitario la mayor parte lo absorbe las partidas destinadas al gasto farmacéutico, siendo el beneficiario último la industria farmacéutica. Por lo que habiendo toda una serie de problemas relacionados con la atención como la masificación, ésta no es la principal partida para invertir. Y por último mencionar como el proceso de privatización de nuestra sanidad y en general de todos los servicios relacionados con el bienestar: educativo, social,... va en incremento. De modo que en nuestro país, hay un gran déficit en protección social y cada vez más desigualdades en salud, educación, protección social,... Además al haber pocos recursos e incrementarse la población y las demandas hay un aumento de la competencia, lo que produce numerosos conflictos no debidos a las características de los grupos inmigrantes y desfavorecidos sino a que no hay un aumento de la inversión en salud.

\section{BIBLIOGRAFÍA}

- ATXOTEGUI J. (2000) Los duelos de la migración: una aproximación psicopatológica y psicosocial. En: Perdiguero E, Comelles JM, editores. Medicina y cultura. Estudios entre la antropología y la medicina. Barcelona: Bellaterra.

- BLÁZQUEZ, M.I.; CASTILLO, S.; MAZARRASA, L.; (2003). "Una experiencia europea de promoción de la salud: la participación comunitaria con comunidades de base del África subsahariana” A tu salud. Marzo.
- BLÁZQUEZ, M.I.; MAZARRASA, L.; (2003) “La promoción de la salud en la población inmigrada". Index de Enfermería, año XII, 42. 34-38.

- Boletín Oficial del Estado. Ley Ogánica 7/1985, de 1 de julio, sobre derechos y libertades de los extranjeros en España. BOE núm 158, 3/7/1985.

- Boletín Oficial del Estado. Real Decreto 239/2000 por el que se el establece el procedimiento para la regularización de extranjeros prevista en la disposición transitoria primera de la Ley Orgánica 4/2000, de 11 de enero, sobre derechos y libertades de los extranjeros en España y su integración social. BOE num 43, 19/2/2000.

- Boletín Oficial del Estado. Real Decreto 142/2001, de 16 de febrero, por el se el establecen los requisitos para la regularización de extranjeros prevista en la disposición transitoria cuarta de la Ley Orgánica 8/2000, de 22 de diciembre, sobre derechos y libertades de los extranjeros en España y su integración social. BOE num 44, 20/2/2001.

- CARBALLO M. GROCUTT M, HADZIHASAJANOVIC A. (1996) Women and Migration: a public health issue. Rapp. Trimest. Statist. Sanit. Mond. 49.; 158-164. Medical migration and inequity of health care. The Lancet, vol. 356. $\mathrm{n}^{\circ}$ 9225: 177. julio 15. 2000.

- CASTILLO RODRÍGUEZ, S. (2002). La salud de la población inmigrante en La atención Primaria de Salud ante la población inmigrante. Madrid, fundación CESM.

- CASTILLO S. (2002). La salud de la población inmigrante. En: Garay J, Fernández C, Garcia M.A, editores. La Atención Primaria de Salud ante la población inmigrante. Madrid: Fundación CESM; p. 3-45.

- INSALUD Atención Primaria del. Datos proporcionados por la Subdirección Provincial (Madrid)

- GONZÁLEZ M., SANZ B., SCHUMACHER R., TORRES AM. (1997) Una aproximación a la identificación de la situación sociosanitaria de la población inmigrante del Area 6 de la Comunidad de Madrid. Ofrim diciembre: 74-93.

- PROYECTO SEPY 1058/98: "La salud de la mujer inmigrante en la Comunidad de Madrid: percepción, accesibilidad y utilización de los servicios sanitarios" financiado por el Instituto de la Mujer. 1998/1999.

- SOLAS GASPAR O. UGALDE A. (1997). Inmigración, salud y políticas sociales. Escuela Andaluza de Salud Pública.

- SHAW M., DORLING D., DAVEY SMITH G., (1999) Poverty social exclusion and minorities. En: Wilkinson R., Marmot M., editors. Social determinants of health. Oxford: Oxford University Press; p. 211-239.

Http://dgei.mir.es/es/general/Anuario2002Presentac ion.pdf

www.ine.es

http://dgei.mir.es/informaciónestadistica. 